\title{
Mobile Augmented Reality at the Hollywood Walk of Fame
}

\section{Thommen Korah*}

\author{
Jason Wither \\ Yun-Ta Tsai
}

Ronald Azuma

Nokia Research Center Hollywood, CA

\begin{abstract}
This work introduces techniques to facilitate large-scale Augmented Reality (AR) experiences in unprepared outdoor environments. We develop a shape-based object detection framework that works with limited texture and can robustly handle extreme illumination and occlusion issues. The contribution of this work is a purely geometric approach for detecting marker-like objects under difficult and realistic outdoor conditions. We demonstrate these techniques for mobile AR experiences by detecting and tracking star-shaped pentagrams embedded in the Hollywood Walk of Fame at $30 \mathrm{~Hz}$ on a Nokia N900 phone.
\end{abstract}

Index Terms: H.5.1 [Information Interfaces and Presentation]: Multimedia Information Systems-Artificial, augmented, and virtual realities; I.4.8 [Image Processing and Computer Vision]: Scene Analysis-Tracking

\section{INTRODUCTION}

The goal of this work is to deploy a large-scale mobile AR experience that exploits outdoor urban landmarks. We use the pentagramshaped markers in Hollywood as an example that is difficult for existing methods [9]. Being on the floor, millions of people walk over them causing wear and appearance changes. Lighting variation with shadows, time of day, glare, and specular highlights pose challenges for appearance-based feature tracking. Detection and tracking in crowded urban streets must deal with extreme occlusion. Appearance-based descriptors like SIFT [8] are insufficient to describe the star shape because of its symmetry and lack of texture. With over 2000 different stars, storing templates for matching is clearly not feasible. Figure 1 shows a result of our detection method with virtual content overlays at the Hollywood Walk of Fame.

We make a number of contributions. At a high level, we are motivated by the challenge of quickly making AR markers and outdoor visual tags from existing landmarks. The preponderance of commercial logos and facility signs in urban scenes make them good candidates as tracking targets. A key design choice was to avoid pixel-based comparisons and use purely geometric methods for detection. Edge segments are extracted and robustly chained to build structural features from an image. These features called $k$-chains capture salient geometric structures. A novelty of our work is the direct use of these features to identify perceptual properties such as symmetry and connectedness. We introduce a hypothesize-andtest procedure that infers the object from a minimal set of $k$-chains based on the shape model. This makes the detection process significantly more efficient for AR than matching to a database of templates. We have built and demonstrated a star tracker using these techniques on a Nokia N900 phone.

Existing fiducial based tracking is reliant on easily thresholded images $[6,5]$. Despite its simplicity and moderate robustness, the environment needs to be instrumented. Wagner [9] and Klein [7]

*corresponding author: thommen.korah@nokia.com

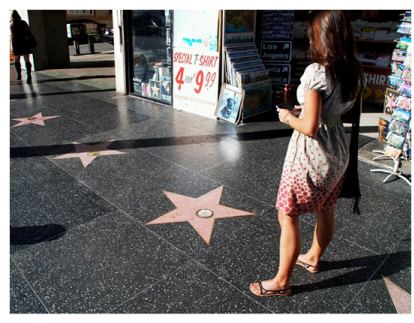

(a)

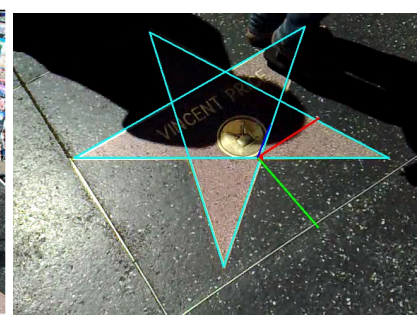

(b)
Figure 1: (a) The Walk of Fame is a popular tourist landmark in Hollywood with over 2000 star-shaped markers embedded on the sidewalk. (b) An example result of our marker detection method on a moderately difficult image.

demonstrated simplified Natural Feature Tracking (NFT) on a mobile phone for AR applications. Lack of texture and symmetric patterns can confuse these algorithms. Shape-based methods [4] that use edges alone can be sensitive to occlusion. There is a rich literature in Computer Vision on wide-baseline image matching using edge and line signatures [1]. Recently, perceptual grouping of line segments has been used successfully in object recognition and detection [3]. These are based on properties such as connectedness, convexity, parallelism, and proximity. Our work is most inspired by Ferrari et al. [3] which used groups of adjacent boundary segments for image matching. They proposed a family of scale invariant local shape features called $k$ adjacent segments ( $k \mathrm{AS}$ ) formed by chains of $k$ connected, roughly straight contour segments. This paper demonstrates techniques to efficiently assemble such features into model shapes for AR.

\section{Image Processing}

Our shape recognition algorithm relies on grouping edges in the image to form a known shape. Two complementary schemes for line segment detection are detailed below. The first method is the Burns line segment extraction algorithm [2] that runs in linear time. The detected segments are robust to lighting variations and cast shadows. Approximately 400-500 lines are detected from a typical $640 \times 480$ image in our dataset (Fig. 2 a).

A more efficient but less stable alternative is color-based segmentation. Although sensitive to illumination and shadow effects, our shape recovery method can tolerate a large amount of noise in the initial processing stage. A 2-color Gaussian mixture model is constructed from manually labeled pixels within and outside the region of interest. The line segments are extracted from the mask by connected components grouping followed by polygon approximation. These resulting points form a polygonal chain; each consecutive set of points is then treated as an individual line segment. The next section describes the process of extracting structural features from the line segments to detect a known shape.

\section{Shape Detection with Chained Edge Segments}

Let $M(\mathbf{x})$ be the model shape with parameters $\mathbf{x}$. Under perspective imaging, the shape $S=\left(p_{1}, p_{2}, \ldots, p_{n}\right)$ is observed as a polygonal 


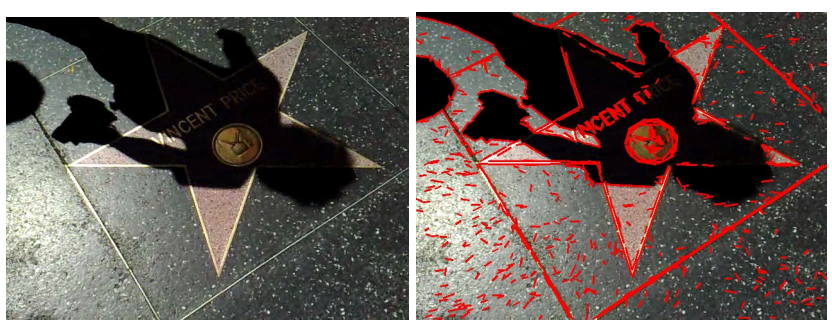

(a)
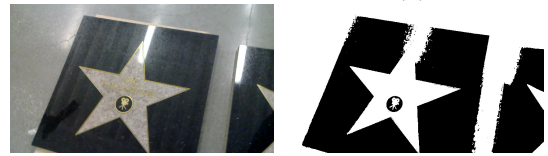

(b)

Figure 2: Image processing methods: (a) An input image and the result of line detection; (b) Lines detected by polygon approximation on a binary mask obtained through a simple and fast color segmentation. Our robust shape recovery techniques can tolerate several false positives in the image processing stage. We also avoid pruning or thresholding out potential lines early in the pipeline in order to pass on as much information as possible into the grouping stages.

chain or series of line segments that connect vertices $p_{i}$. Shape inference involves determining the sequence of points $p_{i}$ under various projective deformations, occlusions, and lighting conditions. The point $p_{1}$ is fixed as the origin and associated with some unique geometric property that allows pose recovery. In our example of the Hollywood stars, the internal corner closest to the circular icon on the star is labeled as the origin.

Ferrari et al. [3] proposed short chains of extracted line segments called $k$ adjacent segments ( $k \mathrm{AS})$ as structural features and match images based on their overall geometric arrangement. A group of $k$ segments is a $k$ AS iff they can be ordered so that the $i$-th segment is connected in the network to the $(i+1)$-th segment for $i \in\{1, k-1\}$. As $k$ grows, $k$ AS can form increasingly complex shape structures: individual segments for $k=1 ; L$ and $T$ shapes for $k=2$ and so on. To distinguish our features from [3], we denote them as $k$ chains. Rather than establishing correspondences between $k$-chains detected in two images, we attempt to directly assemble these features into the target object. The model $M$ guides this process. We argue that this scheme is more efficient for AR applications where the marker is known beforehand.

Chaining two line segments is driven by local constraints. A compatibility function $V\left(l_{i}, l_{j}\right)$ for two neighboring segments is true if and only if one of the endpoints of $l_{i}$ passes near an endpoint of $l_{j}$ and the lines are directed towards each other. We define a distance metric $D\left(l_{i}, l_{j}\right)$ which returns the Manhattan distance between the two closest endpoints of $l_{i}$ and $l_{j}$ if $V$ is true and $\infty$ otherwise. $V$ can be tuned with additional knowledge of shape, but we avoid enforcing constraints too early in the pipeline. $k$ is the number of turns taken when all the line segments are chained together.

Constructing the $k$-chain is similar to path planning algorithms. We use a best first graph traversal that treats each line segment as the vertex of a graph. Chaining is initiated from a node until the number of turns taken exceed $k$. Similar to the results in [3], we have found that pairs of line segment chains with $k=2$ makes the best compromise between discriminative ability and repeatability; low values of $k$ also keeps the detection process efficient. Therefore, all 2-chains are extracted from the image. Since these features might be composed of several segments (chaining two collinear segments will not increase $k$ ), we simplify them to form a descriptor of it's three endpoints $c_{1}, c_{2}, c_{3}$. The middle point $c_{2}$ is the intersection point of the two uniquely oriented line segments in the 2-chain. While most descriptors encode the appearance of pixels around the feature, this descriptor encodes local geometric structure of the line segments in the image.

Model fitting is an iterative process and uses a hypothesize-andtest scheme. Given $N k$-chains extracted from the image, each iteration randomly selects the minimum set of $k$-chains $\mathbf{K}=k_{1}, k_{2}, . ., k_{m}$ required to determine hypothetical model parameters $\mathbf{x}$. For objects exhibiting geometric symmetry, $m$ is likely to be low and in our experiments $N$ is typically 25-30 even from several hundred line segments. The parameters of the model are estimated from this minimal set of $k$-chains and tested for validity. If valid, the approximate model is refined using all the observed line segments extracted around the model shape. The pentagram, for example, is defined by 5 self-intersecting lines for which $m=32$-chains would constitute a minimum set. This technique is similar to methods like RANSAC which iteratively fits a model to observed data with outliers. The combination of top-down shape knowledge and bottom-up geometric features make the detection robust. Using the star as an example, the next section describes some effective methods for fitting model shapes to $k$-chain features.

\section{Star Detection}

The pentagram or 5-pointed star is the simplest regular star polygon. It contains ten points (the five external points of the star, and the five vertices of the inner pentagon) and can be constructed by connecting alternate vertices of a pentagon. It can also be constructed by extending the 5 edges of an internal pentagon until the lines intersect. We exploit both of these properties for shape fitting. For pose, we estimate the location of the 10 corners and make correspondences with "ideal" corners from a fronto-parallel view. Due to symmetry, a unique orientation is determined by labeling the internal corner closest to the circular icon as $p_{1}$ and the remaining 9 corners $p_{2}, \ldots, p_{9}$ in anti-clockwise sequence.

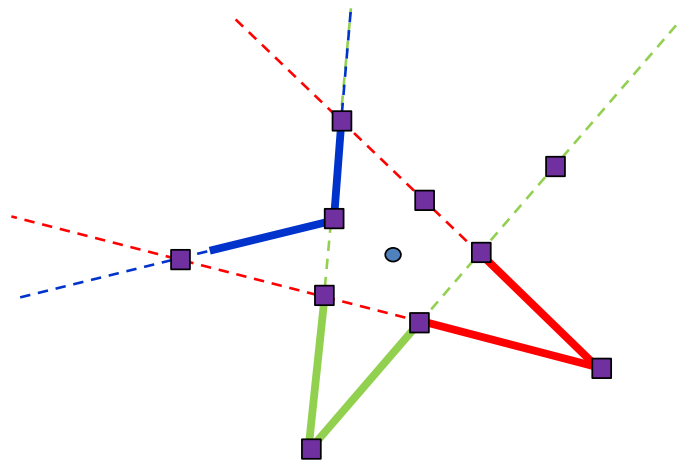

Figure 3: Reconstructing a star from 2-chains. Three such features with 5 unique directions are required to reconstruct the pentagram geometry. Similar to RANSAC-based feature matching, we sample from the list of $k$-chains and check for the star configuration. The thick red, green, and blue lines show a specific example of three 2-chains that adhere to the geometry. The thinner lines show the $k$-chains extended to infinity (only one direction plotted for clarity) and their intersections are plotted as squares.

Given an input image, we extract a set of line segments and detect 2-chains as described above. Each 2-chain consists of two unique directions with its midpoint $c_{2}$ being the intersection of these lines. This bottom-up process results in a number of simple but distinctive structural features; a subset of them must be assembled together by a top-down technique to form the outline of the star.

For 5 unique directions, a minimal sample size of $m=3 k$-chains are required. During each iteration, we pick three $k$-chains and check that there are only 5 unique directions. For every valid set, 


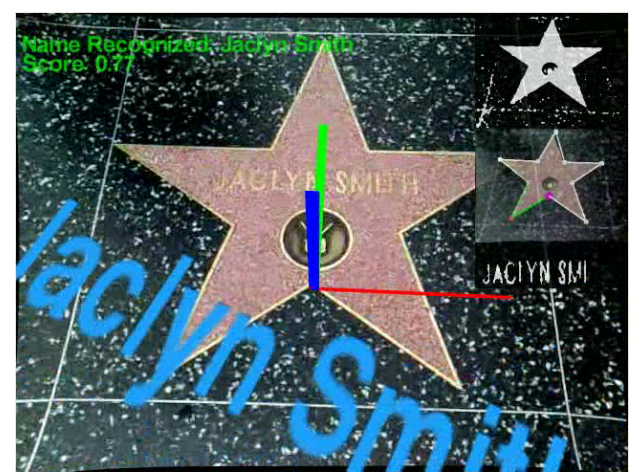

Figure 4: Augmentations on the recognized star.

we compute the intersection $I_{i}$ of all pairs of these line. Figure 3 shows an example of three valid $k$-chains plotted in thick lines colored red, green, and blue. The colored squares are intersections of these line segments when extended to infinity; we check that they have reasonable bounds (parallel lines might stretch to infinity in which case this is not a star). Intersections are required to fall within a rectangular boundary centered on the image and twice its dimensions.

An efficient way to check for the star configuration is to first take the convex hull of intersection points in $I$. For valid sets, the convex hull should result in a pentagon connecting the 5 external corners of the star. We then verify that a putative line from the sampled 2chains connects every other corner of the resulting pentagon. If so, the line intersections and connections adhere to the basic geometry of the star. The five lines $L_{i}$ that form the pentagram are constructed by connecting every alternate vertex of the convex hull. The provisional star corners $p_{1}^{*}, \ldots, p_{9}^{*}$ can then be computed and ordered by intersecting each of the 5 lines to determine internal corners.

A final geometric verification validates that the connections do indeed form a star-shaped pentagram. We avoid thresholding on lengths and angles which are inherently brittle under nonorthogonal views. The cross ratio is an important projective invariant of an ordered 4-tuple of points on a projective line. Given $L_{i}$, let $a, b, c$, and $d$ be the 4 points of intersection on the line. Thus $a$ and $d$ are the external corners, while $b$ and $c$ form the internal points on the edge. The cross-ratio for these 4 collinear points is defined as $\frac{|a c||b d|}{|b c||a d|}$. For pentagrams, the cross ratio of these points is equal to the golden ratio $\phi \approx 1.618$ and is an important property derived from its symmetric shape. Being invariant to perspective viewpoints, our test confirms that the intersections along each of the five lines $L_{i}$ have the correct ratios. To tolerate noise in the sampling procedure, we threshold the ratio to be between 1.5 and 1.7. Once the 10 corners are confirmed to belong to a star, we use all line segments detected along the boundary of the shape to reestimate $L_{i}$. The corners $p_{1}, . ., p_{9}$ are recomputed by intersecting the refined edges.

The final step for pose estimation is to establish correspondences between the estimated corners and a template shape description. Being symmetric, the location of the circular icon is used to identify the origin. We again exploit our chaining technique described in Section 3. Line detection results in several short edge segments on the boundary of the circular arc. Given a series of line segments, we measure the likelihood of it belonging to a circle by determining the length of segments that can be chained together such that turns are taken in only one direction (clockwise or anti-clockwise). For each internal corner, we hypothesize a possible location of the icon as a circle centered on the midpoint of the line joining the internal corner and the star centroid. We then return the origin as the corner with the Maximum Likelihood estimate of containing circular arcs.

\subsection{Name Recognition}

There are over 2000 stars at the Walk of Fame. While shape detection is able to correctly determine camera pose from the currently viewed star, contextual information is augmented by identifying the name engraved on it. We adopted a simple template matching approach to correlate the cropped text region from the rectified star shape to a database of names. The detected star shape is first rectified and scaled to a fixed size; a 256x56 pixel region around the text is cropped. The resulting color image is projected into the Green channel which has higher contrast between the pink marble and gold metal plate. We discovered that the background and foreground text can be approximated as two Gaussian distributions; k-means was used to recover their parameters and produce a binary segmentation of text and non-text pixels. Due to the small size of our database, a template matching approach was sufficient to correctly identify the star. The template matching score is aggregated over individual letters instead of correlating the entire segmented region. Figure 4 shows augmentations performed after name recognition.

\section{EXPERIMENTS AND RESULTS}

We test our star detection algorithm on several videos captured at the Hollywood Walk of Fame using the Nokia N900 phone. The dataset contains sequences of multiple stars taken on different days under a range of conditions. We also tested our algorithms in indoor settings on a custom-built star plaque made of polished marble. Figure 5 gives a synopsis view of star detection and pose estimation under challenging conditions. Mixed and Augmented Reality experiences in outdoor settings must be able to address such unpredictable variation in visibility, image noise, lighting, and cast shadows. Our results demonstrate how a combination of bottomup image processing combined with top-down semantic knowledge can address these issues.

Table 1 shows quantitative results on different videos. Recognition performance is measured as the percentage of frames in which both star boundary and orientation were correctly detected. We get over $90 \%$ detection rate on most of the sequences. The Shape column shows the percentage of frames in which the star boundary was detected correctly (based on manual inspection). The Icon column shows the percentage of frames where both boundary and orientation (location of the emblem) were determined correctly. Note that these results are independently estimated for each image to measure detection performance. During on-line operation, estimation of the icon can be made robust by temporal smoothing. This significantly improves overall detection rate. Finally, among the total of 659 frames in this dataset, there were only 3 false positives.

Our ideas are built on the premise that "if a human can detect the star, the algorithm can". This is possible by exploiting symmetry. As long as any part of the 5 lines are visible in an image, a human can infer the corners of the star. A novelty of the $k$-chain technique is that it can efficiently identify these 5 lines from several hundred line segments. The average number of line segments detected from our data was $N_{l}=475$ and the average number of k-chains extracted was $N_{k}=28$. Exhaustively testing $N_{l}$ choose 5 lines to test for a star shape is not tractable. When picking a minimal sample of $k$ chains for star building, an exhaustive test would require at most $N_{k}$ choose 3 combinations. There is a high probability of finding a good configuration early.

We bench-marked and tested our implementation on the Nokia N900 phone running the Maemo operating system. The N900 is equipped with a $600 \mathrm{MHz}$ ARM Cortex-A8 CPU and 256MB of DDR RAM. Our detection algorithm was integrated into a Mixed Reality Framework (MRF) developed in-house. The framework is developed using the Qt library and enables overlaying virtual content on images streamed from the camera. Image pixels captured by the MRF are at $400 \times 240$ pixel resolution. We use binary mask 

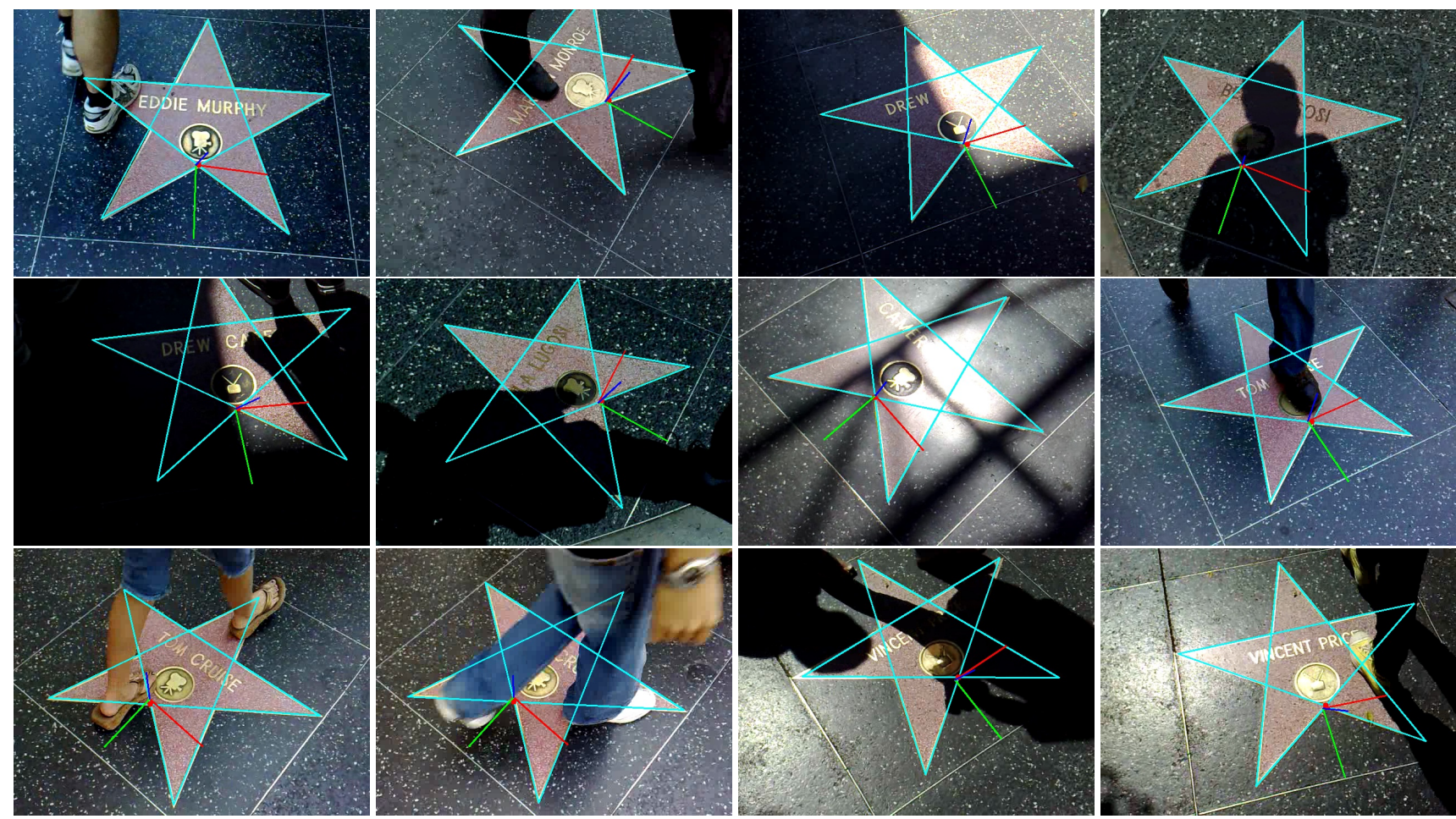

Figure 5: Results of star detection and pose estimation on several example images from our dataset. The results demonstrate robustness to various issues encountered outdoors - shadows, occlusion, and changing illumination. Our approach fits a model star shape to bottom-up features extracted by chaining pairs of adjacent line segments. The symmetric property of the star allows us to infer occluded or noisy edges.

\begin{tabular}{|l|l|l|l|l|}
\hline Sequence & Difficulty & Frames & Shape (\%) & Icon (\%) \\
\hline Eddie Murphy & P & 100 & 95.0 & 95.0 \\
Wesley Snipes & PO & 143 & 95.8 & 90.9 \\
Monroe & PO & 32 & 100.0 & 96.8 \\
Drew Carey & OSL & 60 & 95.0 & 87.0 \\
Vincent Price A & OSG & 59 & 96.6 & 91.5 \\
Vincent Price B & SLG & 152 & 96.0 & 95.4 \\
N900 sequence I & POLG & 59 & 94.9 & 88.1 \\
N900 sequence II & POLG & 54 & 87.0 & 85.1 \\
\hline
\end{tabular}

Table 1: Detection rate for star shape and icon location on different sequences. The tests are performed on frames extracted every 0.5 seconds from the sequence. Each letter in the Difficulty column indicates the following characteristics of the sequence: large perspective changes $(P)$, occlusion $(\mathrm{O})$, shadows $(\mathrm{S})$, changing illumination $(\mathrm{L})$, and specular highlights or glare $(\mathrm{G})$.

segmentation followed by polygon approximation to extract edge segments. Figure 6 shows example results of the detected shape and pose for images captured by MRF on the device. Table 1 also shows detection accuracy for two sequences captured from the N900. Our algorithm achieved a frame rate of $30 \mathrm{~Hz}$ on the device.

\section{Conclusion}

We have described a robust, efficient, edge-based system for detecting markers in outdoor environments. Simple features that capture structural properties of image edges are extracted from the image. They are assembled into a model shape using an efficient hypothesize-and-test framework. The algorithm achieves over $90 \%$ detection rate on challenging images while running at $30 \mathrm{~Hz}$ on a mobile device. Future work involves addressing issues of pose stability, efficiency, and generalization to different markers.
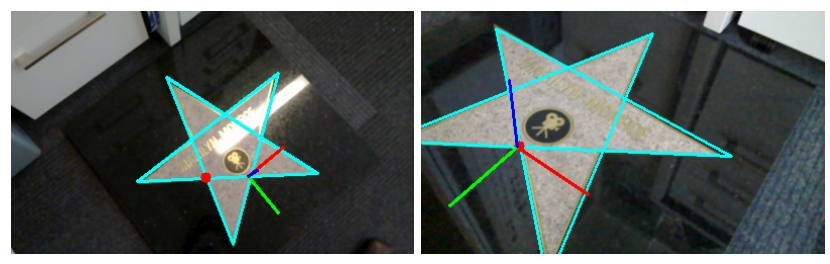

Figure 6: Detected star and estimated pose for images captured by the Mixed Reality Framework running on the Nokia N900. Our algorithm already runs at $30 \mathrm{~Hz}$ on the $\mathrm{N} 900$.

\section{RefEREnCES}

[1] H. Bay, V. Ferrari, and L. V. Gool. Wide-baseline stereo matching with line segments. In Proc. CVPR, 2005.

[2] J. B. Burns, A. R. Hanson, and E. M. Riseman. Extracting straight lines. IEEE Trans. Pattern Anal. Mach. Intell., 8:425-455, 1986.

[3] V. Ferrari, L. Fevrier, F. Jurie, and C. Schmid. Groups of adjacent contour segments for object detection. IEEE Trans. on Pattern Anal. \& Mach. Intell., 30:36-51, 2008.

[4] V. Ferrari, T. Tuytelaars, and L. V. Gool. Markerless augmented reality with a real-time affine region tracker. In Procs. of ISMAR, 2001.

[5] M. Fiala. Artag, a fiducial marker system using digital techniques. In CVPR, 2005.

[6] H. Kato and M. Billinghurst. Marker tracking and hmd calibration for a video-based augmented reality conferencing system. In Proc. of ISMAR, 1999.

[7] G. Klein and D. Murray. Parallel tracking and mapping on a camera phone. In Proc. of ISMAR, 2009.

[8] D. Lowe. Distinctive image features from scale-invariant keypoints. International Journal of Computer Vision, 60:91-110, 2004.

[9] D. Wagner, G. Reitmayr, A. Mulloni, T. Drummond, and D. Schmalstieg. Pose tracking from natural features on mobile phones. In ISMAR, 2008. 